5. Charles V, Charles SX. Primary tuberculosis of the conjunctiva. J Indian Med Assoc 1980;74:74-5.

6. Mehra KS. Tuberculoma of the conjunctiva. Acta Ophthalmol 1964;42:897-9.

7. Cassady JR. Tuberculosis of the conjunctiva. Am J Ophthalmol 1965;60:730-3.

8. Cook CD, Hainsworth M. Tuberculosis of the conjunctiva occurring in association with neighbouring lupus vulgaris lesion. Br J Ophthalmol 1990;74:325-6.

9. Singh I, Chaudhary U, Arora B. Tuberculoma of the conjunctiva. J Indian Med Assoc 1989;87:265-6.

10. Eltringham IJ, Drobniewski F. Multiple drug resistant tuberculosis. Br Med Bull 1998;54:569-78.

David Salas

Shashidhar Murthy

Carrie Champ

Nick Hawksworth

Royal Glamorgan Hospital

Llantrisant CF72 8XR, UK

David Salas, MRCOphth

Eye Department

Royal Glamorgan Hospital

Llantrisant CF72 8XR, UK

Sir,

\section{Necrotising fasciitis of the periorbital region}

Necrotising fasciitis is a life-threatening bacterial infection characterised by necrosis of subcutaneous tissue and underlying fascia, with rapid spread of the infection and inflammation along fascial planes. It rarely involves the face and periorbital region. ${ }^{1}$ Early diagnosis and prompt treatment are essential to prevent the high morbidity and mortality associated with this condition. Although Streptococcus pyogenes is the most frequent isolate, a mixture of facultative and anaerobic bacteria can also lead to this condition. ${ }^{2,3}$ We present a case where prompt diagnosis and instigation of appropriate highdose antibiotics limited the need for extensive debridement.

\section{Case report}

A 37-year-old Caucasian man presented at 4 a.m. to the Eye Casualty. He had allegedly been assaulted and hit with a baseball bat on the right side of his face at 11 a.m.

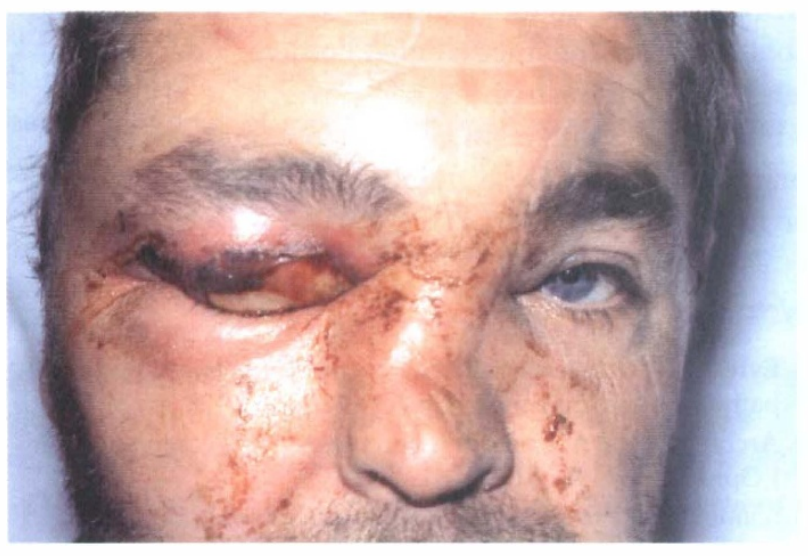

Fig. 1. Photograph showing sloughed necrotic skin of the upper lid and woody swelling on the right side of the face.

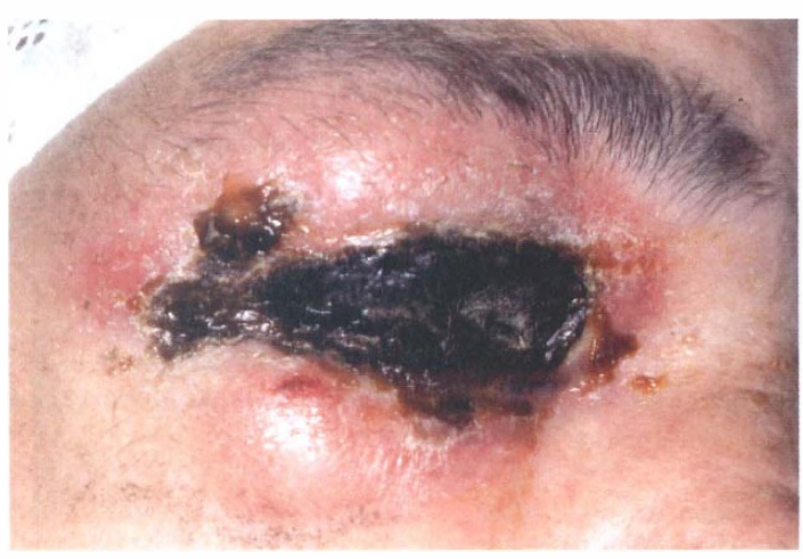

Fig. 2. Photograph showing necrotic eschar formation of the upper lid.

the previous day. He gave a history of brief loss of consciousness. He was a known alcoholic with consumption of 60 units of alcohol per week.

At the time of presentation his temperature was $38.5^{\circ} \mathrm{C}$. He felt unwell and complained of considerable pain on the right side of his face. On examination there was gross swelling of the eyelids and periorbital region on the right side. He also had a superficial laceration on the upper eyelid above the lateral canthus. Eye examination revealed a normal anterior segment. There was no proptosis and ocular movements were normal. The pupillary reactions were equal with no relative afferent pupillary defect. Skull and facial radiographs showed no fractures. His white blood cell count was $20.15 \times 10^{9} / \mathrm{mm}^{3}$ which was suggestive of significant bacterial infection. The plasma urea and electrolytes, liver function tests and clotting factors were normal.

A provisional diagnosis of traumatic haematoma with preseptal cellulitis was made and he was started on intravenous augmentin $1.2 \mathrm{~g}$ t.i.d. After $5 \mathrm{~h}$ he was still unwell and remained pyrexial. He also developed woody swelling of the periorbital region, which extended from the forehead down to the side of his face to the mandibular angle. There was a darker purple area over the upper lid with some flaking of the skin (Fig. 1). At this point a clinical diagnosis of necrotising fasciitis was made. He was started on intravenous benzylpenicillin $1.2 \mathrm{~g}$ 4-hourly, intravenous cefuroxime $1.5 \mathrm{~g}$ t.i.d. and intravenous metronidazole $400 \mathrm{mg}$ t.i.d. A wound and eye swab was taken and microscopy showed Grampositive cocci suggestive of streptococci. An urgent microbiology and dermatology opinion was sought. Twenty-four hours later his temperature had lysed and he felt much better but the dark area in the upper lid had worsened and looked necrotic and crusted (Fig. 2).

The blood culture and wound swab grew Grampositive Group A streptococci after 3 days. He continued to improve clinically. After 7 days of intravenous antibiotics the woody swelling had subsided but the area of necrosis was larger. He therefore underwent debridement of the upper lid on the eighth day. Three days later he had upper lid reconstruction with axillary skin graft. 
In 1952, Wilson coined the currently preferred term necrotising fasciitis' to emphasise the constant feature of necrotic fascia with spread of infection along fascial planes and non-specificity of the bacterial aetiology. ${ }^{4}$

Necrotising fasciitis commonly occurs in the lower extremities, trunk and perineum but rarely involves the face. There is often, though not always, an inciting cause such as a laceration or an infection. Patients frequently have an underlying predisposing condition such as diabetes or alcoholism. ${ }^{4}$ Group A and non-group A Streptococcus and Staphylococcus species are the most common aetiological agents but a mixture of facultative and anaerobic organisms can also lead to this type of infection., ${ }^{2,3}$

The diagnosis depends on clinical awareness and a high index of suspicion about the condition. The signs and symptoms can be nondescript initially but pain is usually out of proportion to clinical signs and patients are significantly unwell. ${ }^{5}$ It is important to commence appropriate antibiotics intravenously once the condition is diagnosed clinically. Samples for bacteriological studies should be obtained before institution of antibiotic therapy. This should include Gram stains of the discharge and aerobic and anaerobic cultures of the wound and blood.

Antibiotic therapy, in particular high-dose intravenous penicillin, has proven efficacy in treating Group A streptococcal infection, ${ }^{6}$ but in some cases of multi-bacillary disease, as in necrotising fasciitis, there is a suboptimal response to penicillin alone. There is emerging evidence of the role of clindamycin in the treatment. ${ }^{7}$ As patients with necrotising fasciitis have toxaemia as a result of streptococcal exotoxins type A, clindamycin, which works by interfering with protein (toxin) production at the ribosomal level, may be helpful in managing the condition even before the organism is killed by beta-lactam antibiotics. ${ }^{8}$

The importance of surgical debridement has long been recognised. ${ }^{9}$ Debridement following involvement of the periorbital region can result in cosmetic

disfigurement and functional problems such as corneal exposure. Reconstruction of the eyelids and skin grafts may be necessary to avoid complications such as cicatricial lid retraction and lagophthalmos.

The mortality in patients with periorbital spread is reported to be $12.5 \%{ }^{1}$ and necrotising fasciitis of the head and neck region has an overall mortality of between $9 \%$ and $31 \%$ in the antibiotic era. ${ }^{10}$ The prognosis is adversely affected by delay in diagnosis and extension of infection from the face to the neck.

The history was misleading in this case because of trauma, but the signs of necrotising fasciitis were unmistakable within $24 \mathrm{~h}$ of injury. It must be emphasised that diagnosis must be made clinically because late diagnosis increases the mortality. We seek to reiterate the importance of early treatment with highdose intravenous antibiotics, including penicillin, which in this case limited the extent of infection and reduced the need for extensive debridement and reconstruction.
1. Kronish JW, Mcleash MW. Eyelids necrosis and periorbital necrotising fasciitis. Ophthalmology 1991;98:92-8.

2. Shindo ML, Nalbone NP, Dougherty WR. Necrotising fasciitis of the face. Laryngoscope 1997;107:1071-9.

3. Giuliano A, Lewis F Jr, Hadley K, et al. Bacteriology of necrotizing fasciitis. Am J Surg 1997;134:52-7.

4. Wilson B. Necrotizing fasciitis. Am Surg 1952;18:416-31.

5. Haywood CT, McGear A, Lowe DE. Clinical experience with 20 cases of Group A streptococcal necrotizing fasciitis and myonecrosis. Plast Reconstr Surg 1999;5:1567-73.

6. Bisno AL, Stevens DL. Streptococcal infections of skin and soft tissues. N Engl J Med 1996;334:240.

7. Brun-Buisson C. The therapeutic approach to necrotizing fasciitis. Ann Dermatol Venereol 2001;128(3-C2):394-403.

8. Norby SR, Norrby-Teglund A. Infections due to group A streptococcus: new concepts and potential treatment. Ann Acad Med Singapore 1997;26:691-3.

9. Wood TF, Potter MA, Jonasson O. Streptococcal toxic shock like syndrome: the importance of surgical intervention. Ann Surg 1993;217:109.

10. Buchanan CS, Haserick JR. Necrotizing fasciitis due to group AB haemolytic streptococci. Arch Dermatol 1970;101:664-8.

B. Pal

S. Evans

R.F. Walters

Department of Ophthalmology

University Hospital of Wales

Cardiff, UK

B. Pal

Leeds General Infirmary

Clarendon Wing

Belmont Grove

Leeds LS2 9NS, UK

Tel: +44 (0)1132432799

Fax: +44 (0)1132926239

e-mail: bishpall7@aol.com

Sir,

Conjunctival metastasis: a rare presentation of bronchial adenocarcinoma

Metastatic involvement of ocular tissues in malignancy is not uncommon; however, tumour deposits in the conjunctiva are extremely rare and indeed few cases are documented in the literature. We report a patient presenting with a rapidly enlarging conjunctival mass found to be an adenocarcinomatous metastasis from an occult pulmonary primary.

\section{Case report}

A 68-year-old man presented with a 10-day history of mild irritation around his left medial canthus associated with a rapidly enlarging painless mass. There were no other complaints or relevant history. On direct questioning he admitted to a loss of appetite and significant weight loss over the preceding month.

Examination revealed the presence of a $18 \mathrm{~mm}$ by $13 \mathrm{~mm}$ solid mass arising from the nasal conjunctiva of the left eye (Fig. 1). The anterior segments were otherwise normal and dilated fundoscopy was unremarkable. Systemic examination revealed the presence of left-sided cervical and supraclavicular lymphadenopathy. 